\title{
Desempenho do Método de Kaczmarz Aleatório com Parâmetros de Relaxação Bem Escolhidos
}

\author{
Leonardo Bravo Estácio * Elias Salomão Helou Neto \\ Instituto de Ciências Matemáticas e de Computação, ICMC - USP \\ Universidade de São Paulo - Programa de Ciências de Computação e Matemática Computacional \\ 13566-590, São Carlos - SP \\ leobravo@icmc.usp.br \\ RESUMO \\ elias@icmc.usp.br
}

O Método de Kaczmarz [6], também conhecido na literatura como Técnica de Reconstrução Algébrica (ART - Algebraic Reconstruction Technique), é um algoritmo iterativo para solução de sistemas lineares $A x=b$. Consiste em uma série de projeções ortogonais alternadas realizadas nos hiperplanos definidos pelas equações do sistema.

Uma variação do método clássico, denominada Método de Kaczmarz Aleatório (RK - Randomized Kaczmarz), apresentada em 2009 por Strohmer e Vershynin em [7] consiste em selecionar os hiperplanos para serem realizadas as projeções ortogonais através de uma probabilidade proporcional à sua norma euclidiana. Sua abordagem pode ser descrita por

$$
x_{k+1}=x_{k}+\lambda \frac{b_{p(i)}-\left\langle a_{p(i)}, x_{k}\right\rangle}{\left\|a_{p(i)}\right\|_{2}^{2}} a_{p(i)}^{t},
$$

sendo que $p(i)$ assume valores entre $\{1, \ldots, m\}$ com probabilidade $\frac{\left\|a_{p(i)}\right\|_{2}^{2}}{\|A\|_{F}^{2}},\|\cdot\|_{F}$ denota a norma de Frobenius, $\|\cdot\|_{2}$ denota a norma Euclidiana ou norma espectral para vetores ou matrizes e $\lambda$ o parâmetro de relaxação. Através desta probabilidade foi provada a taxa exponencial de convergência esperada de

$$
\mathbb{E}\left\|x_{k}-x\right\|_{2}^{2} \leq\left(1-\frac{1}{\hat{\kappa}(A)}\right)^{k}\left\|x_{0}-x\right\|_{2}^{2},
$$

sendo que $\hat{\kappa}(A)=\left\|A^{-1}\right\|_{2}^{2}\|A\|_{F}^{2}$ é o número de condição escalado de $A, x_{0}$ é uma estimativa inicial arbitrária e $\mathbb{E}$ denota a esperança sobre a escolha das linhas. Isto assumindo que a matriz $A$ é de posto completo de modo que $\left\|A^{-1}\right\| \stackrel{\text { def }}{=} \inf \left\{M: M\|A x\|_{2} \geq\|x\|_{2}, \forall x\right\}$ esteja bem definido.

Herman iniciou em 1978 [5] os estudos a respeito do parâmetro de relaxação provando que o algoritmo converge caso $0<\lambda<2$. A partir daí, vários trabalhos [2, 3, 7] comentam que a taxa de convergência do Método de Kaczmarz pode ser acelerada através da seleção de um bom parâmetro de relaxação (vide [4]).

Este parâmetro de relaxação define como será realizada a projeção ortogonal em cada iteração do algoritmo, por exemplo, caso $\lambda=1$ a projeção é realizada de maneira exata e gera um ponto pertencente ao hiperplano selecionado. Porém, caso $\lambda>1$ a projeção ortogonal vai além do ponto pertencente ao hiperplano selecionado (forma sobrerelaxada) e, caso $\lambda<1$ a projeção ortogonal fica em uma posição anterior ao ponto pertencente ao hiperplano selecionado (forma sub-relaxada).

O objetivo deste trabalho foi utilizar nosso método de escolha do parâmetro de relaxação e aplicar ao Método de Kaczmarz Aleatório de Sthromer e Vershynin [7] de forma a acelerar a convergência do algoritmo e comparar o seu desempenho com o Método dos Gradientes Conjugados para Equações Lineares Não Simétricas e Problemas de Mínimos Quadrados (CGLS - Conjugate Gradient Method for Unsymmetric Linear Equations and Least Squares Problems) [1].

De (1) podemos chegar, através de manipulações algébricas, em: $\left\|x^{k+1}-x^{*}\right\|_{2}^{2}=\left\|x^{k-1}-x^{*}\right\|+$ $\left(\lambda^{2}-2 \lambda\right)\left[\left\langle Z_{k-1}, x^{(k-1)}-x^{*}\right\rangle^{2}+\left(-\lambda\left\langle Z_{k-1}, x^{k-1}-x^{*}\right\rangle\left\langle Z_{k}, Z_{k-1}\right\rangle+\left\langle Z_{k}, x^{k-1}-x^{*}\right\rangle\right)^{2}\right]$, dado que $Z_{k}=A_{i(k)} /\left\|A_{i(k)}\right\|_{2}$ foram as linhas selecionadas para o cálculo das iterações do algoritmo. A fim de obtermos (2) e (3), partimos deste resultado e avaliamos primeiro a esperança condicional a respeito da escolha de $Z_{0}, \ldots, Z_{k-1}$ e então a esperança condicional a respeito da escolha de $Z_{0}, \ldots, Z_{k-2}$.

\footnotetext{
*bolsista de mestrado FAPESP
} 
Para uma matriz $A \in \mathbb{C}^{m \times n}$ de posto completo, nossas estimativas do parâmetro $\lambda$ são obtidas por:

$$
\min _{\lambda \in(0,2)}\left(\lambda^{2}-2 \lambda\right)\left(2\|A\|_{F}^{2}+\lambda^{2} \delta-2 \lambda\|A\|_{2}^{2}\right) \quad \text { (2) } \min _{\lambda \in(0,2)}\left(\lambda^{2}-2 \lambda\right)\left(2\|A\|_{F}^{2}+\lambda^{2} \rho-2 \lambda \underline{\sigma}^{2}\right) .
$$

sendo que $\rho=\max _{i=1 \ldots m} \frac{\left\|A a_{i}\right\|_{2}^{2}}{\left\|a_{i}\right\|_{2}^{2}}, \delta=\min _{i=1 \ldots m} \frac{\left\|A a_{i}\right\|_{2}^{2}}{\left\|a_{i}\right\|_{2}^{2}}$ e $\underline{\sigma}$ é o menor valor singular da matriz $A$. Nossos resultados numéricos apontam que a escolha entre as estimativas (2) e (3) se dá a partir da distância $\left\|x_{0}-x^{*}\right\|_{2}$ ou do resíduo $\left\|b-A x_{0}\right\|_{2}$. Caso seja mínima, escolhemos (2), se não, (3).

A minimização (3) é otimista em relação a taxa de convergência do algoritmo e, desta forma, calcula o parâmetro de relaxação $\lambda$ acreditando que o algoritmo terá uma boa convergência. Por outro lado, a minimização (2) é pessimista neste mesmo aspecto. Em geral minimizando (3) obtemos parâmetros $\lambda>1$ e minimizando (2) obtemos $\lambda<1$.

Utilizando o Método RK e o CGLS em um sistema $A x=b$, sendo que $A \in \mathbb{C}^{m \times n}, b \in \mathbb{C}^{m}$, $m=5000, n=500,\|A\|_{F}^{2}=1.29 \times 10^{3}, \underline{\sigma}=19.82,\|A\|_{2}=1.12 \times 10^{3}$, a parte real dos elementos aleatórios da matriz $A$ e do vetor $b$ estão no intervalo $[0,1]$ e a parte imaginária estão no intervalo $[0, i]$. Executamos ambos os algoritmos e analisamos o tempo de convergência do método através do erro $\left\|x_{k}-x^{*}\right\|_{2}$ utilizando, no método de Strohmer, projeções com o valor do parâmetro igual a $\lambda=1$ (projeções exatas do artigo original [7]) e também o valor calculado pela estimativa (3) $\lambda=1.2358$, obtendo a Figura 1.

Analisando a Figura 1 vemos que apesar de reduzir bastante o erro em cada iteração, o algoritmo CGLS se mostrou bem mais custoso do que o Método de Kaczmarz Aleatório de Strohmer. Além disso, vemos o ganho na velocidade de convergência utilizando a estimativa calculada minimizando (3), que é um resultado bastante satisfatório.

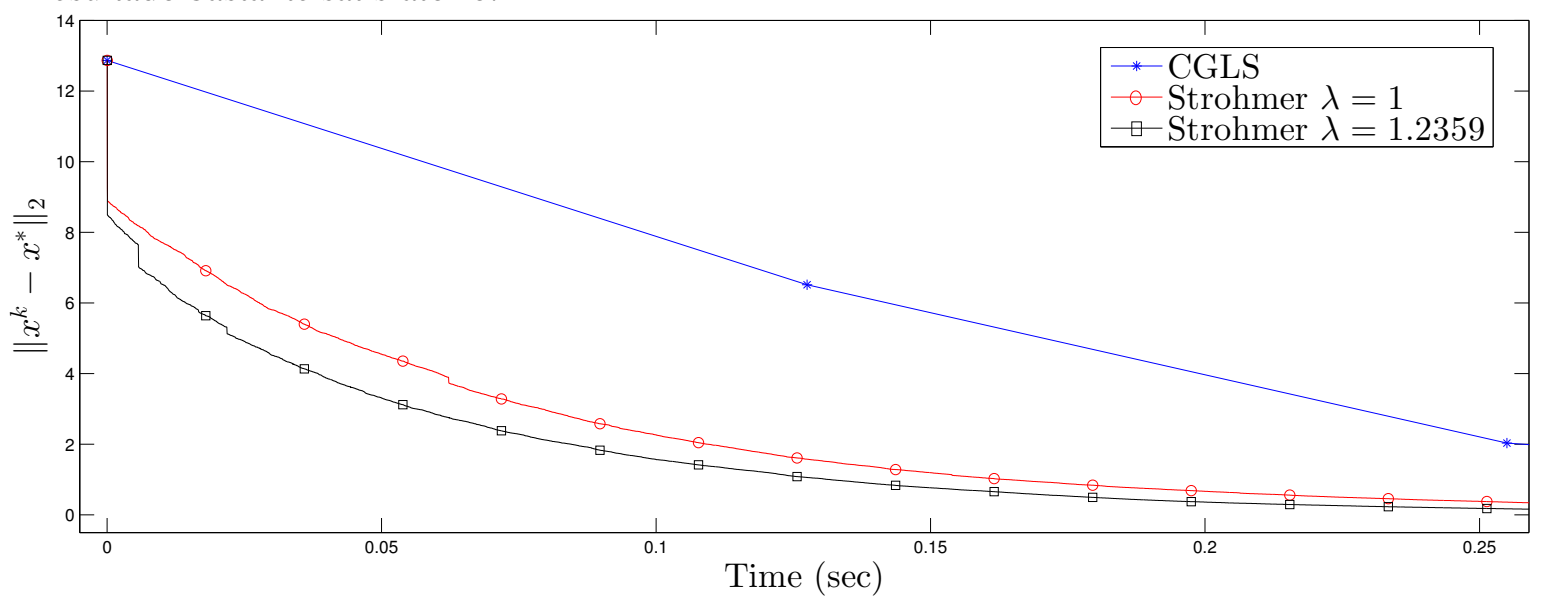

Figura 1: Comparação da velocidade de convergência do Método RK e do Método CGLS

Palavras-chave: Método de Kaczmarz, Parâmetro de Relaxação, CGLS

\section{Referências}

[1] O. Axelsson. Conjugate Gradient type methods for unsymmetric and inconsistent systems of linear equations. Lin. Alg. and its Appl., 29(1):1-16, 1980.

[2] Y. Censor, P. Eggermont, and D. Gordon. Strong underrelaxation in Kaczmarz's method for inconsistent systems. Numer. Math., 41(1):83-92, 1983.

[3] Y. C. Eldar and D. Needell. Acceleration of randomized Kaczmarz method via the JohnsonLindenstrauss lemma. Numer. Alg., 58(2):163-177, 2011.

[4] L. B. Estácio and E. S. Helou Neto. Escolha do parâmetro de relaxação e implementações paralelas do Método de Kaczmarz. In Anais do CMAC (Sudeste), volume 1, pages 701-706, 2013.

[5] G. T. Herman, A. Lent, and P. H. Lutz. Relaxation methods for image reconstruction. Commun. ACM, 21(2):152-158, 1978.

[6] S. Kaczmarz. Angenäherte Auflösung von Systemen linearer Gleichungen. Bull. Acad. Pol. Sci., 35(1):355-357, 1937.

[7] T. Strohmer and R. Vershynin. A randomized Kaczmarz algorithm with exponential convergence. $J$. Fourier Anal. Appl., 15(2):262-278, 2009. 